\title{
El Patentamiento Universitario en Colombia
}

\author{
University Patenting in Colombia
}

\author{
Diana Milena González-Gélvez',Astrid Jaime²
}

\begin{abstract}
El artículo presenta un panorama general del patentamiento que realizan las Universidades en Colombia. Para ello, se realiza un análisis de la información de la base de datos de patentes de la Superintendencia de Industria y Comercio de Colombia desde 1970 hasta 2010. Los resultados permiten observar el estado real del patentamiento Universitario en Colombia, así como identificar las Universidades más exitosas en el proceso de patentamiento y los tiempos reales requeridos para proteger las nuevas creaciones. Así mismo, los resultados son un insumo importante en la determinación de la estrategia de protección que adopten las Universidades Colombianas en cuanto a la protección de sus creaciones.
\end{abstract}

Keywords: propiedad intelectual; universidades; patentes; patentamiento; colombia.

The article presents an overview of university patenting in Colombia. For this propose, we analyze the information about patents, utility models and industrial designs at the Superintendencia de Industria y Comercio's patent database from 1970 to 2010. The results show the real state of university patenting in Colombia, and identify the most successful universities in the patenting process and the actual times required to protect new creations. The results are an important input in determining the protection strategy Colombian Universities should pursue regarding the protection of their new creations.

Keywords: intellectual property; universities, patents; patenting; colombia.

Universidad Industrial de Santander (Colombia). Centro para la Gestión y la Innovación Tecnológica - INNOTEC.

E-mail: 'dianamilenagonzalez@gmail.com, ${ }^{2}$ dirconocim@uis.edu.co

ISSN: 07 I8-2724. (http://www.jotmi.org)

Journal of Technology Management \& Innovation (c) Universidad Alberto Hurtado, Facultad de Economía y Negocios. 


\section{Introducción}

Tradicionalmente, las Universidades han tenido un papel de docencia e investigación. Sin embargo, en las últimas décadas, han sido llamadas a ocupar un rol más directo en el desarrollo económico y a tener un mayor impacto en la sociedad, lo que ha sido llamado la "tercera misión" de las Universidades (Molas-Gallart et al., 2002).

Con el fin de evaluar el papel de las Universidades en su tercera misión, cada vez más indicadores cuantitativos se utilizan para evaluar la calidad y cantidad del trabajo científico generado en las Universidades y su impacto en el Desarrollo Tecnológico y la Innovación (Meyer y Tang, 2007). Wong y Singh (2010), señalan que el patentamiento Universitario ha sido usado como indicador de la participación de las Universidades en la "tercera misión", más allá del rol tradicional de docencia e investigación. Aunque las regalías derivadas del licenciamiento de las patentes universitarias o la frecuencia de creación de spin-offs son indicadores más próximos de la participación universitaria en la comercialización de la tecnología (Siegel et al. 2003; Friedman y Silberman 2003), la información sobre estos no es tan accesible como la información sobre patentamiento.Así mismo, los autores resaltan la dificultad que existe en Colombia no sólo para acceder a la información, sino también para identificar casos de explotación de creaciones, ya que estos son muy escasos. Esta situación dificulta aún más el cálculo de indicadores relacionados con la explotación.

En general, se considera que "las patentes representan un importante vínculo entre la investigación pura y la aplicación industrial, por lo que se convierte en una vía de difusión a través de la cual los científicos pueden orientar sus esfuerzos a las necesidades del mercado y no sólo al propio conocimiento (Kuckartz, 1999)" (Urraca, 2005).

Además, el patentamiento Universitario es una temática que ha tomado mayor importancia en las últimas décadas y Colombia no ha sido la excepción. Las Universidades colombianas han empezado a interesarse en proteger sus creaciones a través del patentamiento, pieza fundamental en el engranaje de la Innovación.

Es por ello que el objetivo de este trabajo es presentar un panorama general sobre el desarrollo a través del tiempo del patentamiento Universitario en Colombia, sin entrar a analizar los asuntos normativos involucrados en el proceso. Para ello, se analizarán las estadísticas sobre las solicitudes y los registros de nuevas creaciones (Patentes, Modelos de Utilidad y Diseños Industriales) de las Universidades Colombianas que, desde los años 80 s, han sido presentadas ante la Superintendencia de Industria y Comercio - SIC -, Órgano Nacional Competente en Materia de PI en Colombia.
El artículo está organizado de la siguiente manera: En un primer capítulo se presenta la metodología utilizada para llevar a cabo el trabajo y en el segundo capítulo se presentan los resultados del análisis, para finalmente dar algunas conclusiones sobre la temática tratada.

\section{Metodología}

En esta sección se presenta la metodología llevada a cabo para realizar el análisis del Patentamiento Universitario en Colombia. En primer lugar, se presenta las formas de PI analizadas; en segundo lugar, la fuente de información y, finalmente, algunos aspectos relevantes tenidos en cuenta al realizar el análisis.

\section{Formas de PI analizadas}

Para realizar el análisis se decidió estudiar las cifras sobre la protección de nuevas creaciones solicitadas por Universidades Colombianas en el país, es decir, patentes de invención, modelos de utilidad y diseños industriales solicitadas por Universidades Colombianas.

Sobre este punto, es importante señalar que para efectos de esta revisión se entenderá por patentes de invención, modelos de utilidad y diseños industriales de Universidades Colombianas aquellas en las que dentro de los solicitantes se encuentre por lo menos una Universidad Colombiana. Por lo tanto, no se tendrán en cuenta las patentes solicitadas por personas naturales 0 instituciones diferentes a las Universidades.

\section{Fuente de Información}

Con el objeto de reunir la información básica sobre las solicitudes de patentes de invención, modelos de utilidad y diseños industriales realizadas por Universidades Colombianas, se consultó la base de datos de Nuevas Creaciones de la Superintendencia de Industria y Comercio (SIC) en Colombia [Portal Web de la SIC para la consulta de nuevas creaciones (patentes, modelos y diseños) http://serviciospub.sic.gov.co/ oparra/serv_57/externas/datospatente. php(Consultado el 2 de abril de 20II.)].

A partir del módulo de consulta de Nuevas Creaciones de la SIC se tuvo acceso a la base de datos de patentes de invención, modelos de utilidad y diseños industriales presentados en Colombia.Allí se obtuvo la siguiente información: año de solicitud, número de expediente, fecha de presentación del trámite ante la SIC, título, disciplina, número de certificado, solicitantes, inventores, estado y fecha de negación, abandono o concesión de las solicitudes de patentes de invención, modelos de utilidad y diseños industriales realizadas por Universidades Colombianas.

ISSN: 07 I8-2724. (http://www.jotmi.org) 
Es importante señalar que el módulo de consulta de Nuevas Creaciones de la SIC es de acceso libre y presenta las solicitudes de protección que han sido publicadas en la Gaceta de la SIC. El módulo de consulta sólo presenta información sobre los trámites que han llegado a la fase de publicación. Concretamente, el trámite de protección establece un periodo de 18 meses entre la fecha de presentación de la solicitud ante la SIC y la publicación en la Gaceta para las solicitudes de patentes de invención y un periodo de 12 meses para las solicitudes de Modelo de Utilidad. De acuerdo con lo anterior, la información obtenida corresponde a las solicitudes de patentes de invención que fueron presentadas ante la SIC antes del 2 de octubre de 2009 o que han sido publicadas en la Gaceta antes de completar los 18 meses y a las solicitudes de Modelo de Utilidad que fueron presentadas ante la SIC antes del 2 de abril de 2010 o que han sido publicadas en la Gaceta antes de completar los 12 meses.

\section{Aspectos relevantes}

Para realizar el análisis de la información obtenida se establecieron los siguientes criterios:

I. La información sobre patentes de invención, modelos de utilidad y diseños industriales fue organizada de acuerdo con la fecha de solicitud del trámite ante la SIC.

2. La fecha de obtención de la información del módulo de consulta de Nuevas Creaciones de la SIC fue el 2 de abril de $20 \mathrm{II}$.

3. Se obtuvieron los registros de las patentes de invención, modelos de utilidad y diseños industriales en las cuales dentro del solicitante se encuentra al menos una Universidad Colombiana.

4. En los casos en que la patente fue solicitada por más de una universidad o por una Universidad y otras entidades se decidió dividir la patente entre el número de solicitantes. Esto con el fin de eliminar la duplicidad de patentes en el estudio. Por ejemplo: Si 2 Universidades solicitaron I patente, se asignó 0,5 patentes a cada universidad.

5. En aquellos casos en que los solicitantes son una Universidad y personas naturales, se decidió contabilizar el grupo de personas naturales como una entidad y dividir la patente. Por ejemplo: Si I Universidad y 3 personas naturales solicitaron I patente, se asignó 0,5 patentes a la universidad.

6. En el caso de las Universidades que cuentan con sedes, se decidió contabilizar las solicitudes de las sedes como una sola entidad correspondiente a la sede central de la Universidad.

\section{Resultados}

A continuación se presentan los resultados de la revisión. En primer lugar, se presentan los resultados obtenidos sobre patentes de invención; en segundo, los correspondientes a patentes de modelo de utilidad y, en tercero, los correspondientes a diseños industriales. En cada caso se presentan las cifras de las solicitudes anuales, el estado de las solicitudes por Universidad y la distribución de solicitudes por disciplina. Finalmente, se presenta la comparación del tiempo de trámite requerido para realizar la protección y las implicaciones de los resultados.

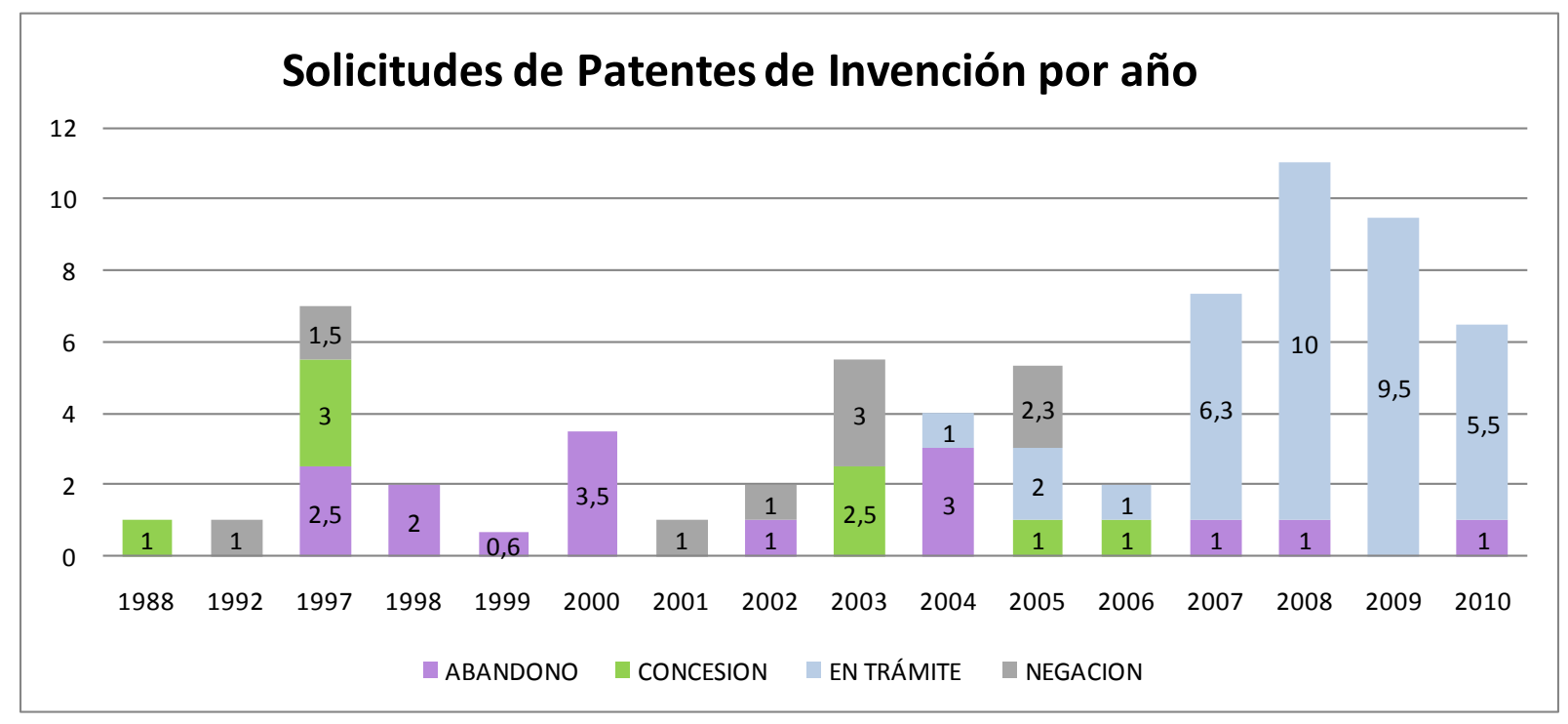

Gráfica I. Solicitudes de Patentes de Invención de acuerdo con el año de presentación de la solicitud ante la SIC discriminando el estado del trámite. Fuente: Elaboración propia a partir de la información consultada en el módulo de Nuevas Creaciones de la SIC.

ISSN: 07 I8-2724. (http://www.jotmi.org)

Journal of Technology Management \& Innovation (c) Universidad Alberto Hurtado, Facultad de Economía y Negocios. 


\section{Patentes de invención}

En relación con las patentes de invención es importante anotar que el módulo de consulta de Nuevas Creaciones de las SIC permite realizar búsquedas a partir de 1970. Sin embargo, el primer registro de una solicitud de patente de invención por una Universidad Colombiana aparece hasta el año 1988. En total se encontraron 69,33 solicitudes. [Los números decimales se originan por aquellas solicitudes que han sido presentadas por varias Universidades, por Universidades y empresas o por Universidades y personas naturales. Estas solicitudes han sido divididas como se explicó en el ítem I.3. aspectos relevantes]

La gráfica I presenta las solicitudes de patentes de invención de acuerdo con el año de presentación de la solicitud ante la SIC discriminando el estado del trámite (abandono, concesión, negación y en trámite):

La gráfica anterior nos permite observar que, a la fecha de la búsqueda ( 2 de abril de $20 \mathrm{II}$ ), se encontraban en trámite solicitudes presentadas desde el año 2004. [Para revisar las gráficas hay que recordar que los números decimales se originan por aquellas solicitudes que han sido presentadas por varias Universidades, por Universidades y empresas o por Universidades y personas naturales. Estas solicitudes han sido divididas como se explicó en el ítem I.3. aspectos relevantes.]

Así mismo, es importante señalar que la gráfica presenta una aparente disminución en el número de solicitudes presentadas en los años 2009 y 2010 . Esto podría deberse a que las solicitudes presentadas a partir de octubre de 2009 no han completado el periodo de 18 meses requeridos para su publicación, momento a partir del cual son visibles en el módulo de consulta de nuevas creaciones de la SIC. Sin embargo, algunas solicitudes presentadas después de octubre de 2009 ya han sido publicadas y otras no han pasado a la fase de publicación. Por esta razón, algunas solicitudes que pueden haber sido presentadas en los años 2009 y 2010 no están incluidas en la gráfica.

En relación con el estado de las solicitudes, la gráfica siguiente muestra el estado de las solicitudes de patentes de invención presentadas entre 1988 y 2010.

De la gráfica anterior llama la atención que el $51 \%$ de las solicitudes de patentes de invención presentadas a partir de 1988 se encuentran en trámite. Así mismo, la gráfica I. indica que las solicitudes que se encuentran en trámite corresponden a solicitudes presentadas a partir del año 2004. Este hecho evidencia el reciente interés de las Universidades Colombianas por obtener patentes de invención.

A pesar del interés creciente de las Universidades colombianas por obtener patentes de invención, se encontró que en 22 años sólo 20 Universidades han presentado alguna vez una solicitud de patente de invención ante la SIC, lo que representa el $25 \%$ de las Universidades del país. [Para el cálculo del porcentaje se utilizó el número de Universidades reconocidas (80) por el Ministerio de Educación Nacional. En Colombia existen 286 IES, de las cuales 80 son reconocidas como Universidades según las estadísticas de Educación Superior emitidas por el Ministerio de Educación Nacional el 23 de Febrero de 20I I. Portal Web: http://www. mineducacion.gov.co/sistemasdeinformacion/I 735/w3-article-2 I2350.html (Consultado el 17 de Mayo de 20I I)]. La gráfica 3. Presenta las solicitudes de patentes de invención de acuerdo con la Universidad solicitante.

\section{Solicitudes Patentes de Invención 1988 - 2010}

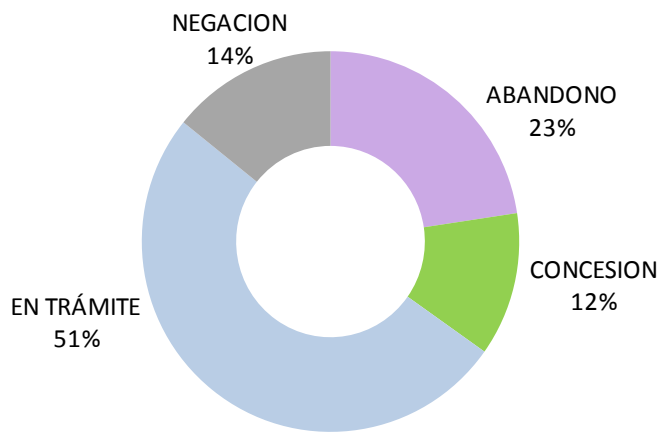

Gráfica 2. Estado de las solicitudes de patentes de invención presentadas entre 1988 y 2010 . Fuente: Elaboración propia a partir de la información consultada en el módulo de Nuevas Creaciones de la SIC.

ISSN: 07 I8-2724. (http://www.jotmi.org)

Journal of Technology Management \& Innovation (c) Universidad Alberto Hurtado, Facultad de Economía y Negocios. 
En la gráfica sobresale la Universidad Nacional de Colombia, por ser la Universidad que ha presentado mayor número de solicitudes y por ser la Universidad con mayor número de patentes de invención concedidas. Así mismo, se encontró que la primera solicitud de patente de invención fue presentada por la Universidad Nacional de Colombia seccional Medellín en el año 1988, constituyéndose en la Universidad con más larga experiencia en el país, 22 años, en la solicitud de patentes de invención. En general, se observa que sólo 4 de las 20 Universidades que han presentado solicitudes de patentes de invención han logrado la concesión de alguna de sus solicitudes.

De otro lado, un aspecto que resulta interesante revisar es la disciplina a la que pertenecen las solicitudes. La gráfica siguiente muestra las disciplinas de las solicitudes presentadas desde 1988, según la clasificación de disciplinas que presenta el módulo de nuevas creaciones de la SIC.

La gráfica anterior indica que el $80,1 \%$ de las solicitudes de patentes de invención, es decir, 55,5 solicitudes presentadas por las Universidades ante la SIC pertenecen a las disciplinas de: Ing. Química (23,3 solicitudes), Ing. Mecánica (17,16 solicitudes) y Biotecnología (I5 solicitudes).

\section{Modelos de utilidad}

En relación con las solicitudes de patente de modelo de utilidad, es importante anotar que el primer registro de una solicitud de patente de modelo de utilidad por una Univer- sidad Colombia ante la SIC aparece hasta el año 1997. En total se encontraron 46,58 solicitudes.

La gráfica 5 presenta las solicitudes de patentes de modelo de utilidad de acuerdo con el año de presentación de la solicitud ante la SIC discriminando el estado del trámite (abandono, concesión, negación y en trámite):

La gráfica 5 indica que a la fecha de la búsqueda ( 2 de abril de $20 \mathrm{II}$ ) se encontraban en trámite solicitudes presentadas desde el año 2006.

Llama la atención que la gráfica no presenta información para el año 2010. Esto se debe a que el módulo de nuevas creaciones de la SIC no presenta información sobre solicitudes presentadas en ese año. Sin embargo, esto podría deberse a que las solicitudes presentadas a partir del 2 de abril de 2010 no han completado el periodo de 12 meses requeridos para su publicación y por ello algunas de ellas no han pasado a la fase de publicación, momento a partir del cual son visibles en el módulo de consulta de nuevas creaciones de la SIC.

Una vez revisada la cantidad de solicitudes, a continuación se muestra el estado de las solicitudes de patentes de modelo de utilidad presentadas entre 1997 y 2010.

En contraste con la gráfica 2 que muestra que el $51 \%$ de las solicitudes de patentes de invención presentadas entre 1988 y 2010 están en trámite, la gráfica 6 indica que, sólo el 14\%

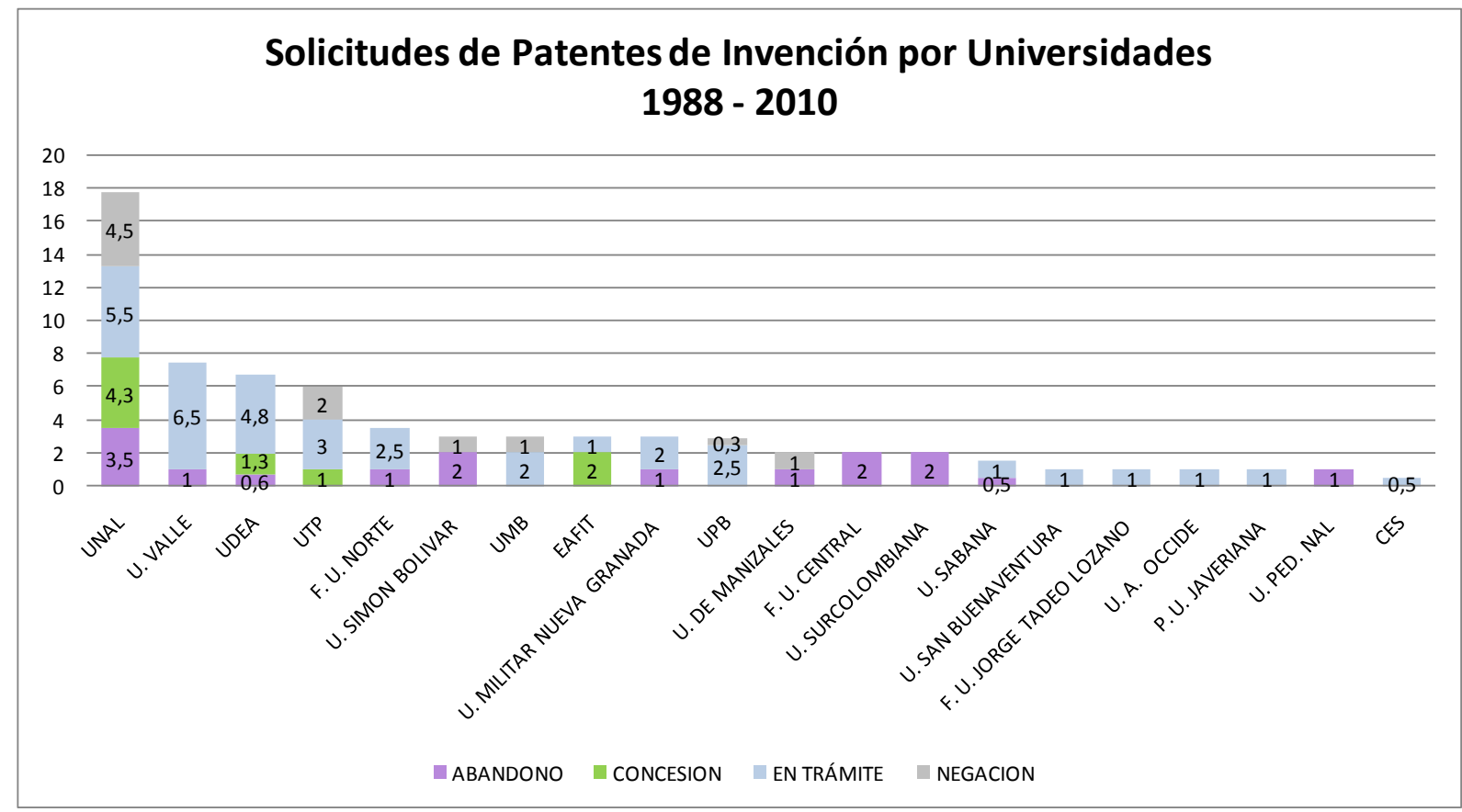

Gráfica 3. Solicitudes de patentes de invención presentadas ante la SIC entre 1988 y 2010 de acuerdo con la Universidad solicitante. Fuente: Elaboración propia a partir de la información consultada en el módulo de Nuevas Creaciones de la SIC.

ISSN: 07 I8-2724. (http://www.jotmi.org)

Journal of Technology Management \& Innovation (c) Universidad Alberto Hurtado, Facultad de Economía y Negocios. 


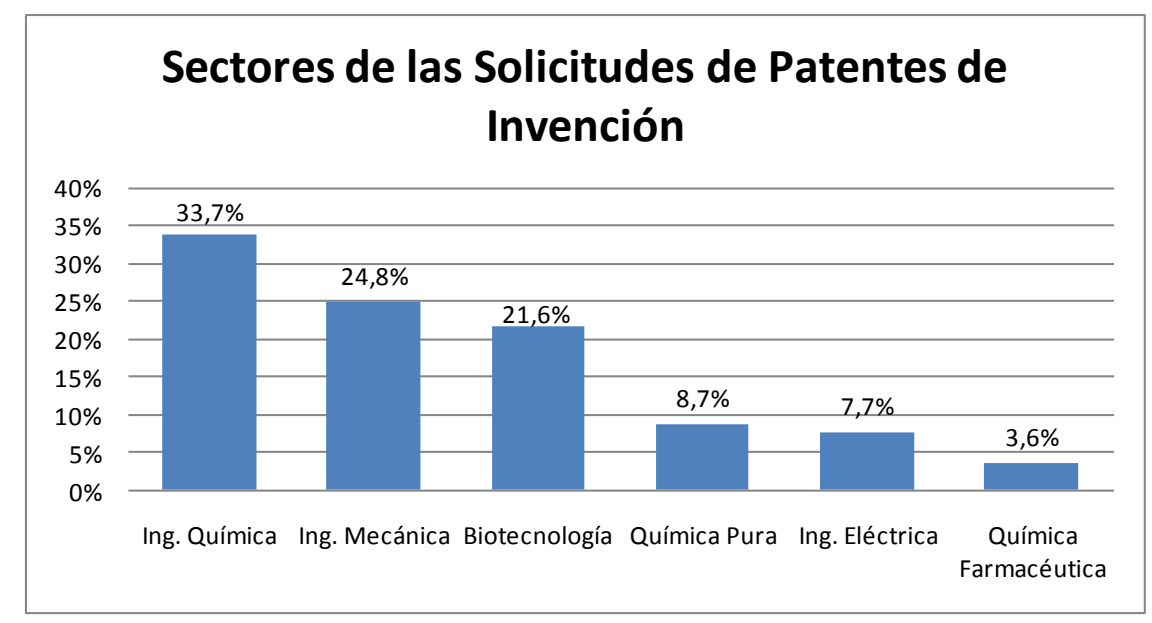

Gráfica 4. Disciplina de las solicitudes de patentes de Invención presentadas desde 1988. Fuente: Elaboración propia a partir de la información consultada en el módulo de Nuevas Creaciones de la SIC

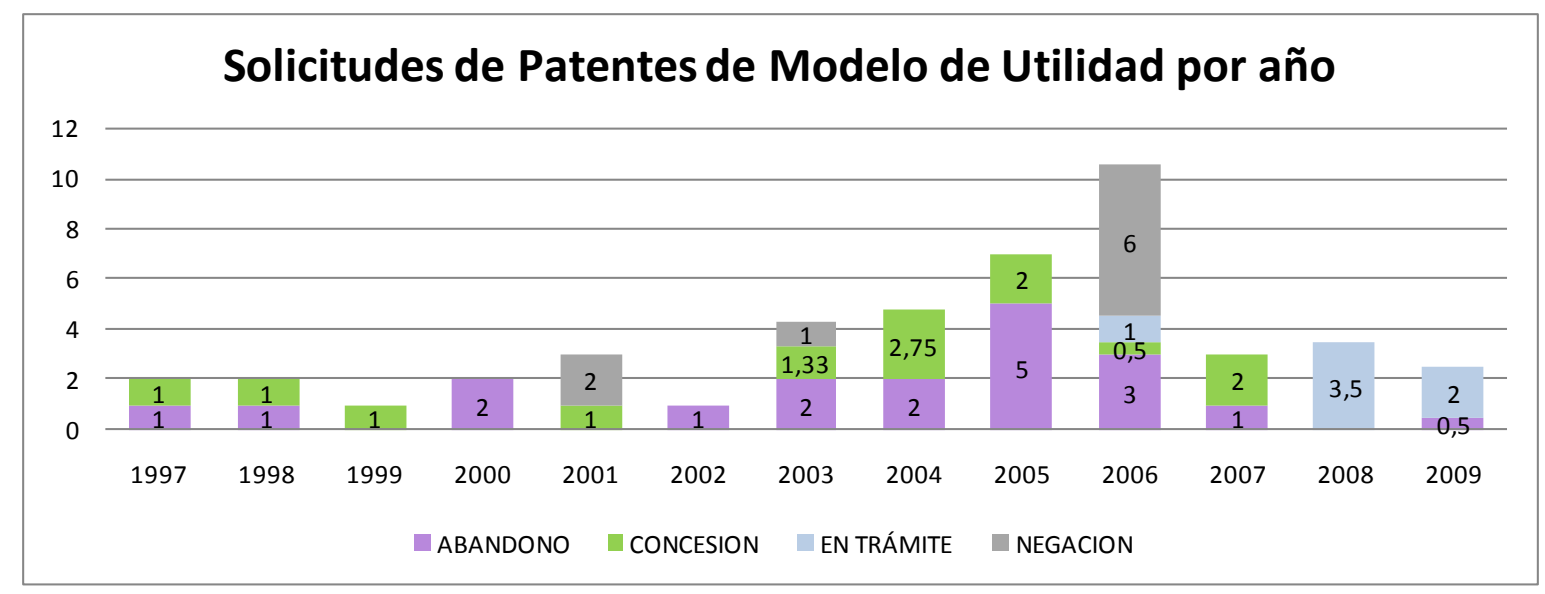

Gráfica 5. Solicitudes de patentes de modelo de utilidad de acuerdo con el año de presentación. Fuente: Elaboración propia a partir de la información consultada en el módulo de Nuevas Creaciones de la SIC

\section{Solicitudes Patentes de Modelo de Utilidad}

$1997-2010$

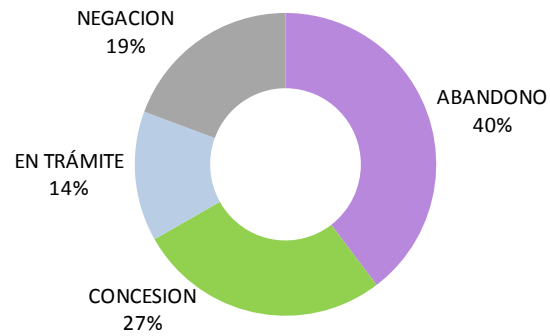

Gráfica 6 Estado de las solicitudes de Patentes de Modelo de Utilidad presentadas entre 1997 y 2010 . Fuente: Elaboración propia a partir de la información consultada en el módulo de Nuevas Creaciones de la SIC.

ISSN: 07 I8-2724. (http://www.jotmi.org)

Journal of Technology Management \& Innovation (c) Universidad Alberto Hurtado, Facultad de Economía y Negocios. 
de las solicitudes de Modelo de Utilidad se encuentran en trámite. Sin embargo, sobresale el hecho de que el $40 \%$ de las solicitudes de modelo de utilidad han sido abandonadas.

En relación con las solicitudes de patentes de modelo de utilidad presentadas por las Universidades, la gráfica 7 presenta la distribución entre las diferentes universidades.

De acuerdo con la gráfica anterior, la Universidad Nacional de Colombia presenta el mayor número de solicitudes de patentes de modelos de utilidad, así como el mayor número de solicitudes concedidas. En general, sólo 7 de las 15 Universidades que han presentado solicitudes de patentes de modelos de utilidad han logrado la protección de alguna de sus solicitudes.

En relación con la disciplina a la que pertenecen las solicitudes de patentes de modelo de utilidad, la gráfica siguiente presenta las disciplinas de las solicitudes presentadas desde 1997 de acuerdo con la clasificación utilizada por el módulo de nuevas creaciones de la SIC.

A partir de la gráfica anterior se observa que el $72,6 \%$ de las solicitudes de patentes de modelo de utilidad están relacionadas con la Ing. Mecánica.

\section{Diseños industriales}

En relación con las solicitudes de protección de los diseños Industriales es importante anotar que el primer registro de solicitud de protección de diseños industriales por una Universidad Colombiana ante la SIC se realizó sólo hasta el año 2005. La gráfica 9, presenta la distribución de solicitudes de protección de diseños Industriales de acuerdo con el año de solicitud.

La gráfica permite observar que el número de solicitudes de Diseños Industriales es bastante menor que en el caso de patentes de invención y de modelo de utilidad.Así mismo, se observa que durante los años 2006 y 2009 las Universidades no presentaron solicitudes.

Una vez revisada la cantidad de solicitudes, a continuación se muestra la gráfica 10 , relacionada con el estado de las solicitudes. Esta gráfica nos permite observar que los diseños industriales presentan la tasa de concesión más alta, pues el $67 \%$ de las solicitudes han sido concedidas (es decir, 8 de las 12 solicitudes presentadas han sido concedidas). Así mismo, llama la atención que el trámite de protección parece ser bastante corto, ya que no se encuentran solicitudes en trámite.

En relación con la distribución de solicitudes de acuerdo con la Universidad solicitante, la gráfica II muestra que sólo 3 Universidades han presentado solicitudes de protección de Diseños Industriales. Así mismo, se observa que la Universidad Nacional de Colombia ha logrado la protección de todas sus solicitudes de protección de Diseños Industriales.

En general, el bajo número de solicitudes de protección, la

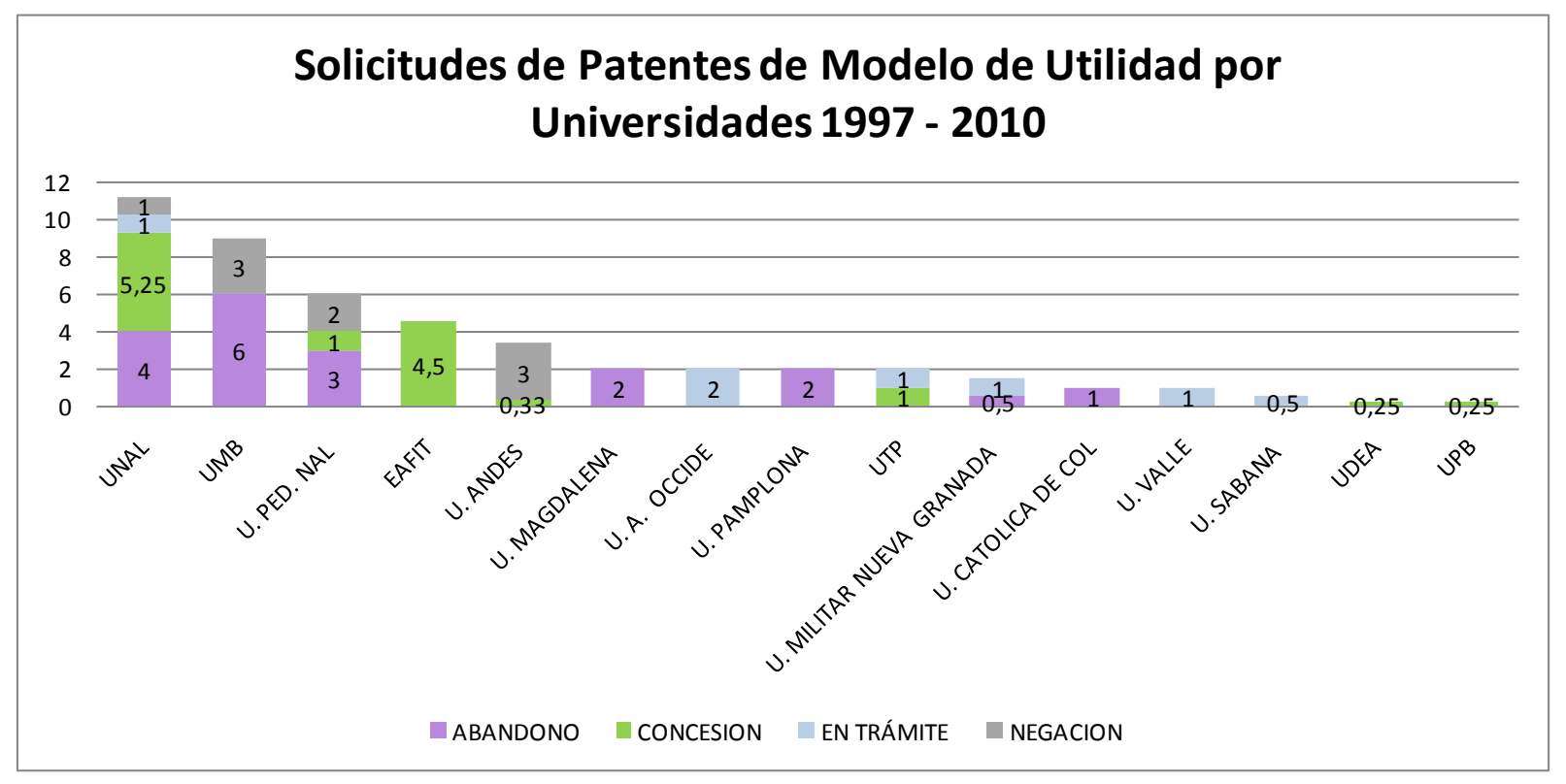

Gráfica 7 Solicitudes de Patentes de Modelo de Utilidad presentadas por Universidades entre 1997 y 20I0. Fuente: Elaboración propia a partir de la información consultada en el módulo de Nuevas Creaciones de la SIC.

ISSN: 07 I 8-2724. (http://www.jotmi.org)

Journal of Technology Management \& Innovation (c) Universidad Alberto Hurtado, Facultad de Economía y Negocios. 
discontinuidad en sus solicitudes (en los años 2006 y 2009 no se presentaron solicitudes universitarias) y el hecho de que la primera solicitud se haya realizado hasta el año 2005, evidencian que la protección de diseños industriales es una práctica que apenas empieza a ser utilizada por las Universidades.Adicionalmente, su alta tasa de concesión la convierte en una figura interesante para todas aquellas Universidades que cuenten con creaciones que puedan ser protegidas por este tipo de registro.

Finalmente y una vez analizadas las solicitudes de patente de invención, modelo de utilidad y diseños industriales en los ítems anteriores, se advierte la gran cantidad de solicitudes abandonadas, negadas o que se encuentran en trámite durante algunos años, lo cual da una idea de la complejidad y del tiempo que toma el trámite de protección. Por ello, conocer los tiempos reales de trámite resulta fundamental para todas aquellas Universidades interesadas en utilizar estas formas de protección. Teniendo en cuenta lo anterior, se presenta a continuación la revisión de los tiempos de cada uno de estos trámites.

\section{Duración de los trámites}

Durante la revisión de los trámites de protección se observó que existen diferencias importantes en los tiempos de trámite de las solicitudes de patentes de invención, modelo de utilidad y diseños industriales. Por ejemplo, en el caso de las solicitudes de diseños industriales, el tiempo mínimo de respuesta de la SIC fue de 6 meses y el máximo fue de 14 meses, a diferencia de las solicitudes de patente de invención, en las cuales el tiempo mínimo de respuesta de la SIC fue de 34 meses y el máximo fue de 81 meses. Para aclarar este aspecto se presenta a continuación la tabla I., la cual compara el tiempo de trámite de Patentes de Invención, Modelos de Utilidad y Diseños Industriales de acuerdo con el estado de la solicitud (concesión, abandono y negación) de las solicitudes presentadas desde 1988 hasta 2010.

La tabla I, muestra claramente que el tiempo promedio para obtener la concesión de una patente de Invención es de 4,5 años, mientras que en los modelos de utilidad es de 4 años y en diseños industriales de 0,8 años. Así mismo, el tiempo

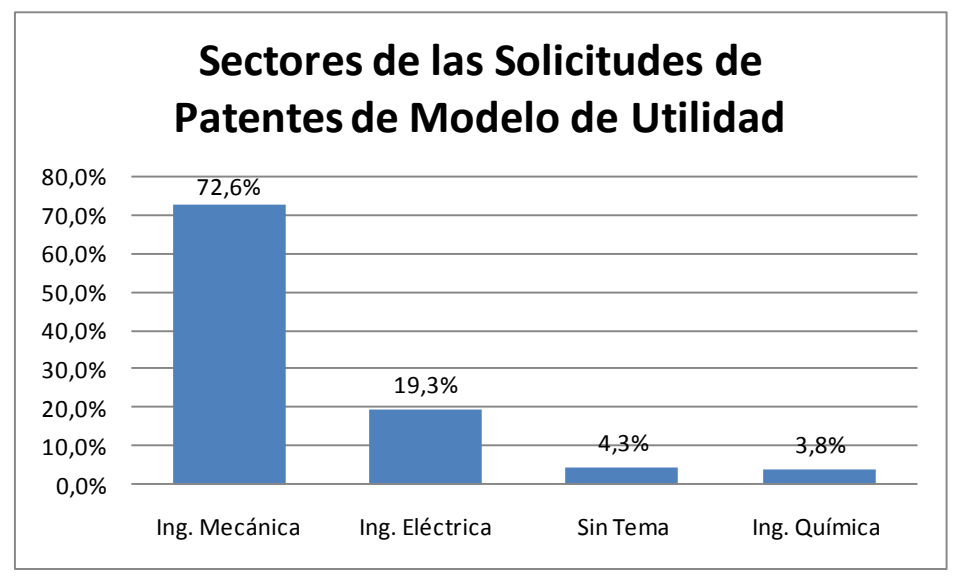

Gráfica 8. Clasificación de las solicitudes de patentes de modelo de Utilidad de acuerdo con la disciplina. Fuente: Elaboración propia a partir de la información consultada en el módulo de Nuevas Creaciones de la SIC.

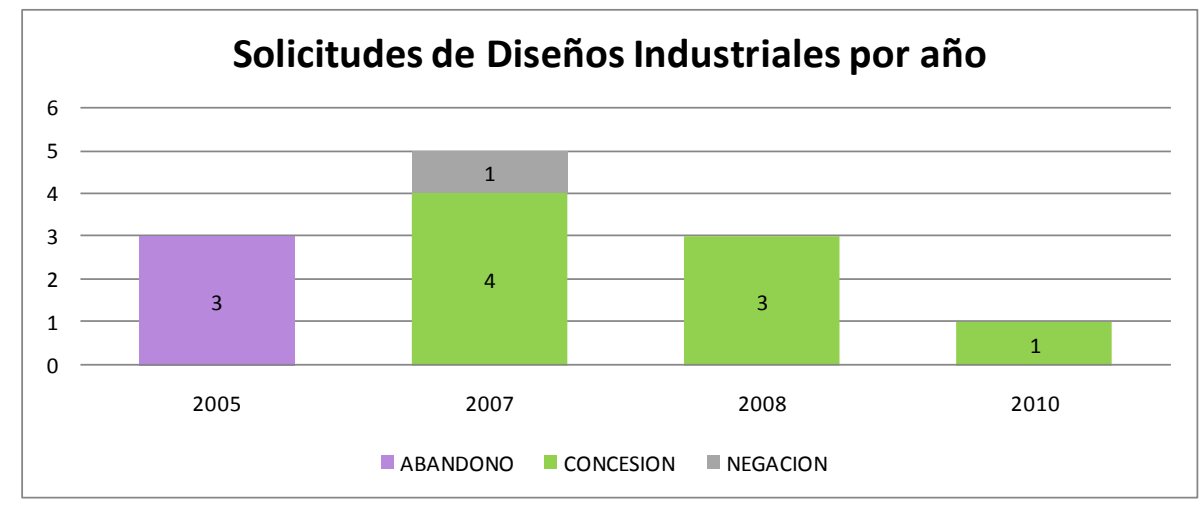

Gráfica 9. Solicitudes de protección de Diseños Industriales de acuerdo con el año de solicitud. Fuente: Elaboración propia a partir de la información consultada en el módulo de Nuevas Creaciones de la SIC.

ISSN: 07 I8-2724. (http://www.jotmi.org)

Journal of Technology Management \& Innovation (C) Universidad Alberto Hurtado, Facultad de Economía y Negocios. 
mínimo para obtener respuesta de solicitudes de Patentes de Invención y Modelos de Utilidad es aproximadamente 3 años. De otro lado, la gráfica corrobora que el trámite más corto es la protección de diseños industriales, que dura aproximadamente I año.

Además, llama la atención que las solicitudes de patentes de invención que fueron negadas presentan un tiempo promedio de respuesta de $5, I$ años, lo que resulta superior al tiempo promedio de concesión (de 4,5 años). En el caso de las patentes de modelo de utilidad el tiempo promedio de respuesta a las solicitudes negadas fue de 3,7 años, lo que resulta inferior al tiempo promedio de concesión (de 4,0 años).

En relación con las solicitudes que fueron abandonadas, se observa que los abandonos empiezan a presentarse desde el inicio de las solicitudes. Sin embargo, el promedio de abandonos se da alrededor de los 2 años para las patentes de invención y alrededor del año y medio para las patentes de modelo de utilidad.

En general, la comparación de los tiempos de trámite de las patentes de invención, modelo de utilidad y diseños industriales permite tener una idea del tiempo real que toma el trámite de protección, ya sea para obtener la concesión o una negación. En todo caso, conocer estos tiempos resulta fundamental para todas aquellas Universidades interesadas en utilizar estas formas de protección. Las universidades deben ser conscientes que el trámite durará varios años, en los cuales probablemente sea necesario responder solicitudes de la SIC en tiempos predefinidos, para lo cual resulta de suma importancia realizar un constante seguimiento a las solicitudes para no caer en abandonos.

Adicionalmente, el interés creciente de las Universidades en el patentamiento, es notorio por el incremento reciente en las solicitudes de protección presentadas ante la SIC.

\section{Solicitudes de Diseños Industriales 2005 - 2010}

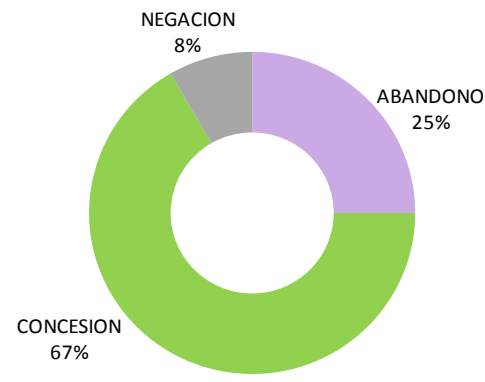

Gráfica 10. Estado de las solicitudes de protección de Diseños Industriales entre 2005 - 20I0. Fuente: Elaboración propia a partir de la información consultada en el módulo de Nuevas Creaciones de la SIC.

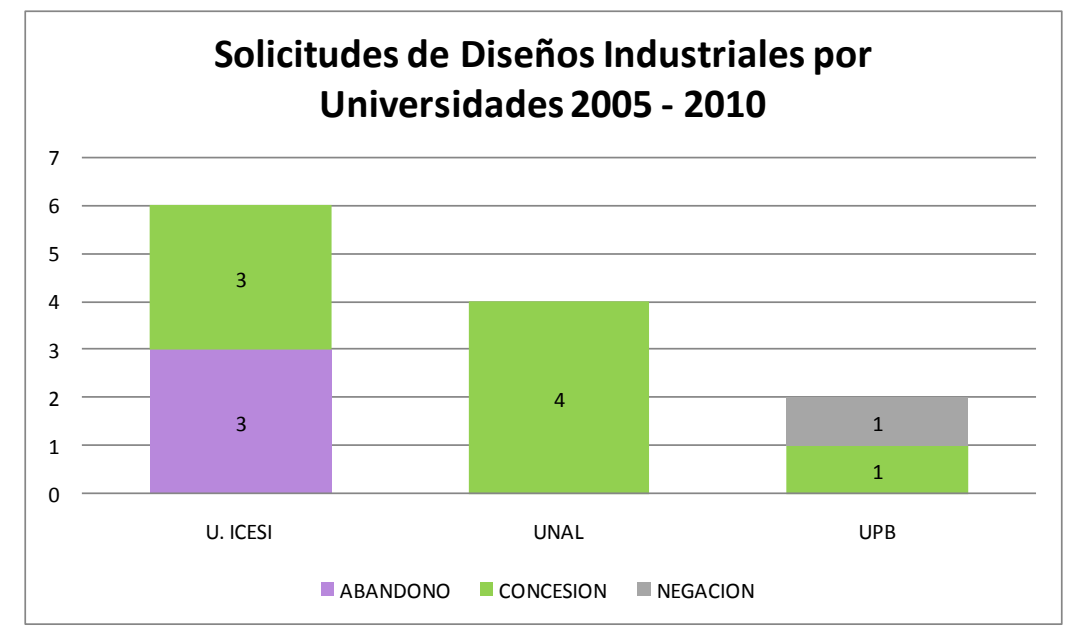

Gráfica II. Solicitudes de Diseños Industriales de acuerdo con la Universidad solicitante. Fuente: Elaboración propia a partir de la información consultada en el módulo de Nuevas Creaciones de la SIC.

ISSN: 07 I8-2724. (http://www.jotmi.org)

Journal of Technology Management \& Innovation (C) Universidad Alberto Hurtado, Facultad de Economía y Negocios. 
Otro aspecto importante es la inclusión de indicadores relacionados con la PI dentro de los sistemas de medición del desempeño de las Universidades Colombianas. Por ejemplo, en el modelo de indicadores de gestión de las Universidades públicas propuesto por el Ministerio de Educación Nacional y el Sistema de Universidades Estatales - SUE, desarrollado para evaluar el desempeño de las Universidades públicas y utilizado para realizar la distribución de recursos adicionales de que trata el artículo 87 de la ley 30 de 1992, se incluyen dos indicadores llamados "Solicitudes de registro de derechos de Propiedad Intelectual presentados por la Universidad ante la Autoridad Competente" y "Registros Otorgados de Propiedad Intelectual", los cuales comprenden en su cálculo las patentes de invención, modelos de utilidad, diseños industriales, obtentores vegetales y esquemas de trazado de circuitos integrados.

Estos aspectos hacen necesario que las Universidades establezcan estrategias para la gestión de su $\mathrm{Pl}$ y que al fijar las metas de sus indicadores de PI tengan en cuenta que el proceso de protección es complejo y prolongado, de forma que se establezcan metas realistas.

\section{Implicaciones de los resultados}

Una vez realizado el análisis de la información sobre solicitudes y registros de nuevas creaciones (Patentes de invención, Modelos de Utilidad y Diseños Industriales) de la base de datos de Patentes de la SIC, es preciso abordar a continuación algunas implicaciones de la información analizada: Por una parte, el análisis de la información sobre patentamiento Universitario evidencia que el trámite de protección es bastante prolongado (aproximadamente 3 años, como mínimo, para obtener respuesta en patentes de invención y modelo de utilidad), por lo cual es recomendable que la SIC realice un estudio de sus tiempos, $y$ en la medida de lo posible, realice comparaciones con instituciones encargadas de los trámite de protección en otros países y analice las alternativas para reducir los tiempos de trámite.

De otro lado, el interés creciente de las Universidades en la temática, el bajo número de Universidades que han solicitado trámites de protección y las altas tasas de negación y abandono en algunos trámites, hacen necesario que la SIC continúe realizando capacitaciones sobre el trámite de protección en todo el país, vinculando, en especial, a las Universidades, de forma que éstas aprendan la forma de adelantar dichos trámites y comiencen o incrementen la protección de sus creaciones. Además, es necesario fortalecer aquellas universidades que ya han iniciado procesos de protección, de forma que se evite caer en causales de abandono, que les puedan impedir ser exitosas en dichos procesos.

En relación con la base de datos, se reconoce y resalta el esfuerzo de la SIC por mantener una base de datos actualizada, de libre acceso y que presenta la mayoría de información sobre los trámites, lo cual brinda transparencia al proceso. Sin embargo, el mecanismo de búsquedas es limitado y podría ser objeto de mejoras, ya que, por ejemplo, no permite la obtención de reportes que faciliten el desarrollo de análisis como los presentados en este trabajo. En el caso de este estudio, fue necesario realizar la consulta de cada uno de los trámites solicitados por cada Universidad con el fin de construir una base de datos propia que facilitara la realización de cálculos y comparaciones.

\begin{tabular}{|l|l|l|l|l|}
\hline \multirow{4}{*}{ CONSECION } & & & \\
\cline { 2 - 5 } & LIPO DE SOLICITUD & 5,6 & 5,4 & 1,2 \\
\cline { 2 - 5 } & Promedio: & 4,5 & 4,0 & 0,8 \\
\cline { 2 - 5 } & Límite Inferior: & 3,4 & 2,8 & 0,5 \\
\cline { 2 - 5 } & Límite Superior: & 6,8 & 4,8 & 0,9 \\
\cline { 2 - 5 } & Promedio: & 5,1 & 3,7 & 0,9 \\
\cline { 2 - 5 } & Límite Inferior: & 2,9 & 3,3 & 0,9 \\
\cline { 2 - 5 } & Límite Superior: & 6,7 & 4,1 & 0,7 \\
\cline { 2 - 5 } & Promedio: & 2,2 & 1,3 & 0,7 \\
\cline { 2 - 5 } & Límite Inferior: & 0,3 & 0,2 & 0,7 \\
\hline
\end{tabular}

Tabla I. Comparación del tiempo de trámite de protección de acuerdo con el estado de las solicitudes presentadas desde 1988 hasta 2010 (tiempo en años). Fuente: Elaboración propia a partir de la información consultada en el módulo de Nuevas Creaciones de la SIC.

ISSN: 07 I8-2724. (http://www.jotmi.org)

Journal of Technology Management \& Innovation (c) Universidad Alberto Hurtado, Facultad de Economía y Negocios. 


\section{Conclusiones}

El artículo constituye la primera iniciativa de presentar el estado real del patentamiento Universitario en Colombia, permitiendo identificar aspectos importantes para la gestión de la PI en las Universidades Colombianas.

Una vez revisadas las estadísticas de patentamiento Universitario en Colombia se observa que la Universidad con mayor número de solicitudes presentadas es la Universidad Nacional de Colombia con 33 solicitudes en 22 años, de las cuales ha obtenido la concesión de 13,5 solicitudes. Esta cifra es un indicador importante para fijar las metas de protección de las Universidades que estén interesadas en proteger sus creaciones, ya que evidencia que el trámite de protección es un proceso complejo y prolongado que en la mayoría de los casos no termina en la concesión de la protección solicitada.

En cifras generales, se encontró que 25 Universidades Colombianas han solicitado la protección de sus creaciones a través de patentes de invención o de modelo de utilidad y de diseños industriales, lo que representa el $31,25 \%$ de las Universidades del país. De éstas, sólo 8 Universidades han obtenido la protección de alguna de sus creaciones, es decir, el 10\% de las Universidades Colombianas. [Para el cálculo de los porcentajes se utilizó el número de Universidades reconocidas (80) por el Ministerio de Educación Nacional. En Colombia existen 286 IES, de las cuales 80 son reconocidas como Universidades según las estadísticas de Educación Superior emitidas por el Ministerio de Educación Nacional el 23 de Febrero de 20I I. Portal Web: http://www. mineducacion.gov.co/sistemasdeinformacion/1735/w3-article-2 12350.html (Consultado el I7 de Mayo de 20I I)].

Esta situación, sugiere que en Colombia, al igual que se concluyen en los estudios de Meyer et al. (2005) y de Baldini (2006), las patentes universitarias están altamente concentradas en algunas instituciones.

De otro lado, las estadísticas muestran que cada día las Universidades están más interesadas en proteger sus creaciones, lo cual es importante para gestionar la PI universitaria y lleva a la necesidad de estudiar la evolución de esta dinámica. En este sentido, y a medida que la práctica del patentamiento se incremente en las Universidades del país, será recomendable realizar estudios similares a los de Henderson (1998) y Sampat et al. (2003), en los cuales se utilice el análisis de citaciones de patentes como indicador de la calidad de las mismas.

De igual manera, resulta fundamental realizar futuros estudios que midan el impacto de las políticas colombianas en el nivel de patentamiento de las Universidades, similares a los realizados por Mowery et al. (200I), Mowery et al. (1999) y (Shane, 2004), en los cuales estudiaron el efecto de la ley Bayh Dole.Así mismo, es aconsejable realizar comparaciones entre diferentes países latinoamericanos de manera similar a los estudios realizados en Europa por Geuna y Nesta (2006) y comparaciones a nivel regional como el estudio realizado por Azagra-Caro et al. (2006).

En relación con el tiempo requerido para realizar el trámite de protección, las Universidades interesadas en proteger sus creaciones deben ser conscientes que el proceso de protección es bastante prologado, aunque el tiempo de trámite depende del tipo de protección que se realice. En general, es posible decir que el tiempo mínimo de respuesta en Patentes de Invención y Modelos de Utilidad es de aproximadamente 3 años.

En este mismo sentido, se recomienda a la SIC realizar un estudio de los tiempos de trámite de sus procesos de concesión, en especial el relacionado con las patentes de invención y modelo de utilidad, y compararlos con los tiempos de trámite de las Autoridades Nacionales Competentes en otros países, ya que un tiempo prolongado del trámite de protección afecta la competitividad de las creaciones. Además, analizar la conveniencia de implementar mecanismos como el programa "Track One" de la Oficina de Patentes y marcas de los Estados Unidos (The United States Patent and Trademark Office - USPTO), el cual promete un tiempo de trámite de 12 meses. [Para conocer sobre el programa "Track one" se sugiere consultar en el portal web de la USPTO o el siguiente enlace http://www.uspto. gov/patents/init_events/Track_One.jsp (Consultado el 24 de julio de 20/2)]

Una lección importante en relación con las solicitudes abandonadas es que las Universidades que presenten sus solicitudes de protección deben ser bastante cuidadosas durante el trámite y deben dar respuesta a los requerimientos en los tiempos establecidos para evitar que sus solicitudes sean declaradas abandonadas. [Para conocer las situaciones que ocasionan la declaratoria de abandono, se sugiere consultar la Decisión Andina 486 de 2000. Especialmente, los artículos: $9,12,13,39,44,93,120$ y 144]. Así mismo, se considera importante realizar futuros estudios en los cuales se analicen las causas de los abandonos con mayor profundidad.

En relación con las disciplinas de mayor patentamiento Universitario, se observó que en el país el $80 \%$ de las solicitudes de patentes de invención pertenecen a las disciplinas de: Ing. Química (33,7\%), Ing. Mecánica $(24,8 \%$ y Biotecnología $(21,6 \%)$ y que el $72,6 \%$ de las solicitudes de modelo de utilidad pertenecen a Ingeniería Mecánica. Estos resultados contrastan con los hallados por Lissoni (2012) en Europa, quien señala que el patentamiento universitario europeo

ISSN: 07 I8-2724. (http://www.jotmi.org)

Journal of Technology Management \& Innovation (c) Universidad Alberto Hurtado, Facultad de Economía y Negocios. 
está concentrado en sectores como el farmacéutico y biotecnológico, seguido por el sector químico y de materiales, seguido por las mediciones e instrumentación científica, y la Ingeniería Eléctrica y Electrónica.

En cuanto a los porcentajes de concesión, abandono y negación de solicitudes, se observa que el porcentaje de concesión más alto se presenta en Diseños Industriales (67\%) y el más bajo en Patentes de Invención (27\%). El porcentaje solicitudes negadas más alto se presenta en las Patentes de Invención. (32\%) y el porcentaje más alto de abandonos se presenta en Modelos de Utilidad (52\%). Esta situación podría estar relacionada con el grado de complejidad de las creaciones cuya protección se solicita. Sin embargo, sería necesario realizar algunos estudios con el fin de validar la relación. Finalmente, es importante resaltar que este trabajo evidencia el aumento del interés de las Universidades por la PI en las últimas décadas. Así mismo, es un insumo importante en la determinación de la estrategia de protección que adopten las Universidades Colombianas en cuanto a la protección de sus nuevas creaciones ya que será un referente importante en la determinación de las metas de los indicadores de protección y permitirá priorizar las formas de PI que la Universidades decidan proteger.

\section{Agradecimientos}

Los autores agradecen el apoyo recibido de la Universidad Industrial de Santander y el programa ALFA III de la Unión Europea a través de la financiación del proyecto de investigación PILA NETWORK Red de Propiedad Intelectual e Industrial en Latinoamérica, dentro del cual se desarrolla este trabajo. Sin embargo, el contenido de este artículo es de responsabilidad de sus autores y no refleja las opiniones oficiales de los financiadores. Así mismo, agradecen a la Superintendencia de Industria y Comercio por permitir el acceso libre al módulo de consulta de nuevas creaciones.

\section{Referencias}

PANDEY, S., Sharma, R.R.K. (2009). Organizational factors for exploration and exploitation. Journal of Technology Management and Innovation, 4(I), 48-58.

AZAGRA-CARO, J.N., Yegros-Yegros, A., Archontakis, F., (2006). What do university patent routes indicate at regional level?. Scientometrics, 66, 219-230.

BALDINI, N., (2006). The Act on inventions at public research institutions: Danish universities' patenting activity. Scientometrics, 69, 387-407.

FRIEDMAN, J. and Silberman, J., (2003). University technology transfer: Do incentives, management, and location matter?. Journal of Technology Transfer, 28, (I), p. 17-30. In:

WONG. P.K., and Singh. A., University patenting activities and their link to the quantity and quality of scientific publications. (2010) Scientometrics, 83, (I), 27I-294.

GEUNA,A., and Nesta, L.J.J., (2006). University patenting and its effects on academic research: The emerging European evidence, Research Policy, 35, 790-807.

HENDERSON, R., Jaffe, A., Trajtenberg, M., (1998). Universities as a source of commercial technology: A detailed analysis of university patenting 1965-1988, The Review of Economics and Statistics, 80, 119-127. In:

SAMPAT, B., Mowery, D., Ziedonis, A. (2003). Changes in university patent quality after the Bayh-Dole Act: A re-examination., International Journal of Industrial Organization, 21, |37|-|390.

KUCKARTZ, M., (1999). Commercial Explotation of Academic and Scientific Research Inventions - a new duty for patent information. World Patent Information, 21, 27-29. In:

URRACA RUIZ., A., (2005). Patentes y Función Pública Universitaria en Europa: Mitos y Realidades. Revista Brasileira de Inovação, 4, (2), 39|-423.

MEYER,M., Du Plessis,M., Tukeva, T., Utecht, J.-T., (2005). Inventive output of academic research: a comparison of two science systems. Scientometrics, 63, 145-161.

MEYER, M., and Tang, P., (2007). Exploring the "value" of academic patents: IP management practices in UK universities and their implications for Third-Stream indicators. Scientometrics, 70, (2), 4I5-440.

ISSN: 07 I8-2724. (http://www.jotmi.org) 
MOLAS-GALLART, J., Salter, A., Patel P., Measuring Third Stream Activities: Final Report, the Russell Group in the Russell Group of Universities. Brighton, SPRU, University of Sussex, 2002. In: MEYER, M., Tang, P., (2007). Exploring the "value" of academic patents: IP management practices in UK universities and their implications for Third-Stream indicators. Scientometrics, 70, (2), 4I5-440.

MOWERY, D., Nelson, R., Sampat, B., Ziedonis, A., (1999). The effects of the Bayh-Dole Act on US university research and technology transfer: an analysis of data from Columbia University, the University of California, and Stanford University. In: Macho-Stadler, I., Pérez-Castrillo, D., Veugelers, R., (2007). Licensing of university inventions: The role of a technology transfer office International Journal of Industrial Organization, $25,483-510$

MOWERY, D., Nelson, R., Sampat, B., Ziedonis, A., (200I). The growth of patenting and licensing by U.S universities: an assessment of the effects of the Bayh Dole act of 1980, Research Policy, 30, 99-119.

SAMPAT, B., Mowery, D., Ziedonis, A. (2003). Changes in university patent quality after the Bayh-Dole Act: A re-examination., International Journal of Industrial Organization, 21, |37|-1390.

SHANE, S., (2004) Encouraging university entrepreneurship? The effect of the Bayh-Dole Act on university patenting in the United States. Journal of Business Venturing, |9, |27-15 |

SIEGEL, D. S.,Waldman, D.A.,Atwater, L. E., Link,A. N. (2003). Commercial knowledge transfers from universities to firms: Improving the effectiveness of university-industry collaboration. Journal of High Technology Management Research, 14, (I), I I I-I33. In:WONG. P.K., and Singh.A., (20I0). University patenting activities and their link to the quantity and quality of scientific publications. Scientometrics, 83,(I), 27I-294.

URRACA RUIZ., A., (2005). Patentes y Función Pública Universitaria en Europa: Mitos y Realidades. Revista Brasileira de Inovação, 4, (2), 39I-423.

WONG. P.K., and Singh. A., (2010). University patenting activities and their link to the quantity and quality of scientific publications. Scientometrics, 83, (I), 27I-294. 